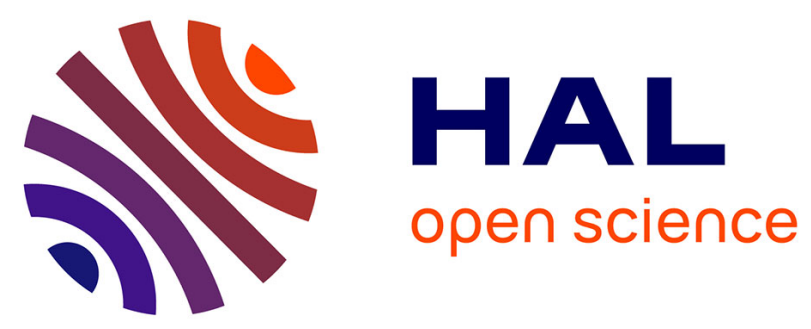

\title{
COMPORTEMENT SOUS SOLLICITATIONS DYNAMIQUES D'ACIERS PRÉSENTANT DE LA PLASTICITÉ INDUITE PAR TRANSFORMATION
}

J. Diani, J. Ponsonnaille, P. Bensussan, M. Berveiller

\section{- To cite this version:}

J. Diani, J. Ponsonnaille, P. Bensussan, M. Berveiller. COMPORTEMENT SOUS SOLLICITATIONS DYNAMIQUES D'ACIERS PRÉSENTANT DE LA PLASTICITÉ INDUITE PAR TRANSFORMATION. Journal de Physique IV Proceedings, 1991, 01 (C3), pp.C3-77-C3-83. 10.1051/jp4:1991310 . jpa-00249901

\section{HAL Id: jpa-00249901 https://hal.science/jpa-00249901}

Submitted on 1 Jan 1991

HAL is a multi-disciplinary open access archive for the deposit and dissemination of scientific research documents, whether they are published or not. The documents may come from teaching and research institutions in France or abroad, or from public or private research centers.
L'archive ouverte pluridisciplinaire $\mathbf{H A L}$, est destinée au dépôt et à la diffusion de documents scientifiques de niveau recherche, publiés ou non, émanant des établissements d'enseignement et de recherche français ou étrangers, des laboratoires publics ou privés. 


\title{
COMPORTEMENT SOUS SOLLICITATIONS DYNAMIQUES D'ACIERS PRÉSENTANT DE LA PLASTICITE INDUITE PAR TRANSFORMATION
}

\author{
J.M. DIANI* J.Ph. PONSONNAILLE * , P. BENSUSSAN"* et \\ M. BERVEILLER*** \\ "DGA, Etablissement Technique Central pour 1'Armement, 16 bis \\ av. Prieur de la côte d'or, F-94114 Arcueil, France \\ ** DGA/DAT, BP 24, F-92211 st Cloud cedex; France \\ *** Laboratoire de Physique et de Mécanique des Matériaux, \\ université de Metz, Faculté des Sciences, Ile du Saulcy, F-57045 \\ Metz, France
}

\begin{abstract}
The mechanical behaviour of a TRIP steel under static and dynamic loadings is presented. Microscopic investigations (optical microscopy and TEM) were carried out to establish correlations between microstructure and macroscopic behaviour. Differences between static and dynamic behaviour are shown to be due to the elevation of temperature during dynamic Hopkinson tests.

Résumé - L'étude du comportement static et dynamique d'un acier TRIP est présentée. Nous nous sommes placés à l' échelle microscopique (microscopie optique et en transmission) pour corréler la microstructure et le comportement macroscopique. Nous avons montré que les différences entre le comportement statique et dynamique étaient dues à l'élévation de température pendant l'essai aux barres d'Hopkinson.
\end{abstract}

\section{Introduction}

L'optimisation des aciers à haute résistance passe aujourd'hui par une meilleure compréhension des mécanismes de déformation et des transformations microstructurales se produisant lorsque ces matériaux sont soumis à des chargements dynamiques.

On constate par exemple que ces "micro" effets peuvent avoir une influence importante sur le comportement "macroscopique" de tels aciers. La figure 1 illustre cette remarque; elle représente en effet le comportement de deux aciers - 45NCDV16 et Z30MCDS1010 ausformé (taux de corroyage de 3,8 à une température de $450^{\circ} \mathrm{C}$ ) - soumis à la même sollicitation dynamique lors d'un essai de compression aux barres d'Hopkinson. Le premier possède une limite d'écoulement élevée mais peu de "moyens" microstructuraux pour se déformer ce qui a pour conséquence une rupture prématurée par localisation. Le second possède une limite d'écoulement plus faible ce qui se traduit ici par des dislocations plus mobiles autorisant une déformation plus grande que dans le cas précédent. On constate en outre une remontée de la courbe quand la déformation atteint $5 \%$, ce qui n'est pas explicable par un mécanisme du type plasticité classique (mouvement de dislocations). Une étude métallurgique révèle l'apparition d'une seconde phase métallique plus dure que la structure initiale et qui induit un relèvement de la limite d'écoulement ainsi q'une déformation supplémentaire sans endommagement. En effet ce type de transformation de phase se propage à la vitesse du son dans le matériau et joue pleinement aussi bien sous sollicitations statiques qu'à grande vitesse de déformation. 


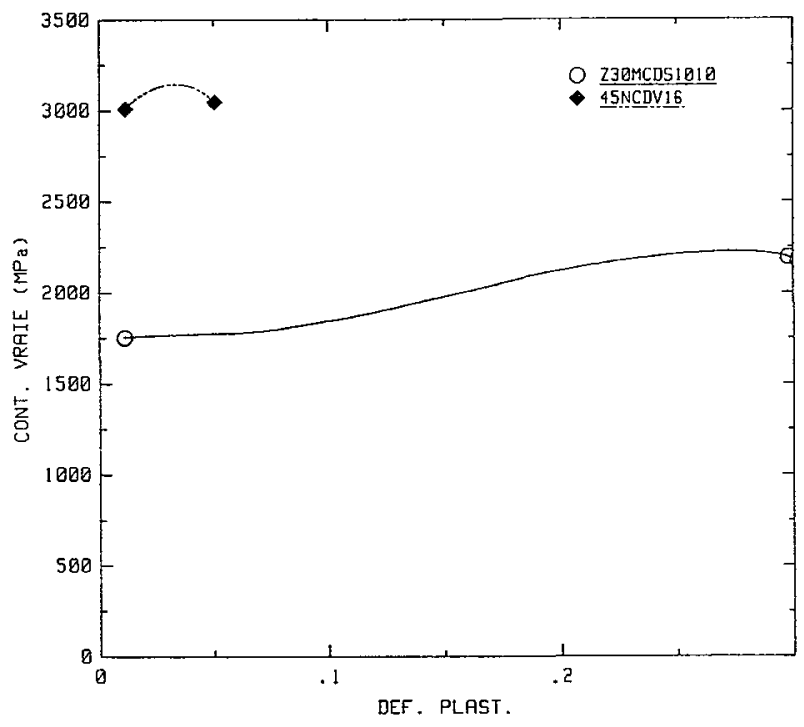

Figure 1 : compression dynamique de deux aciers haute résistance (45NCDV16 et Z30MCDS1010) à $-50^{\circ} \mathrm{C}$.

Dans ce qui suit nous présentons pour l'acier Z30MCDS1010 une étude du comportement macroscopique statique et dynamique à la lumière des mécanismes microscopiques de déformation.

\section{Etude du comportement statique}

L'acier étudié dans la suite est de type Z30MCDS1010 de composition donnée au tableau 1.

Tableau 1 : composition de l'acier Z30MCDS1010 (en \% pondéral).

$\begin{array}{lllll}\text { C } & \text { Mn } & \text { Cr } & \text { Mo } & \text { Si } \\ 0,3 & 10 & 10 & 4 & 2\end{array}$

A la différence du matériau présenté sur la figure 1 l'acier étudié dans la suite n'a pas subi d'austéniformage (à $>\mathrm{M}_{\mathrm{d}}$ ). Son traitement thermomécanique est classique ; à partir d'un rond de diamètre $130 \mathrm{~mm}$ et forgé à $1100^{\circ} \mathrm{C}$ on obtient un plat de $80 \mathrm{~mm}$ d'épaisseur qui est ensuite laminé jusqu'à une épaisseur de $20 \mathrm{~mm}$ (sans élargissement notoire) à la même température et refroidi à la température ambiante. Ce plat est ensuite trempé à l'eau à partir d'une température de $1100^{\circ} \mathbf{C}$. On obtient donc une austénite métastable d'une taille de grains de l'ordre de $40 \mu \mathrm{m}$.

\section{1) Essais de traction en température}

Sur la figure 2 nous présentons des essais de traction à rupture effectués en statique à une vitesse de déformation de $10^{-4} \mathrm{~s}^{-1}$ pour différentes températures.

Pour ces essais nous avons dosé la phase $\alpha^{\prime}$ formée pour la déformation à rupture lors de l'essai à l'aide d'un magnétomètre saturant. Les échantillons ont été prélevés à un endroit quelconque du fût de l'éprouvette car il n'y a pas apparition de striction pour les températures pour lesquelles le taux de martensite est significatif $\left(\mathrm{T}<100^{\circ} \mathrm{C}\right)$. 


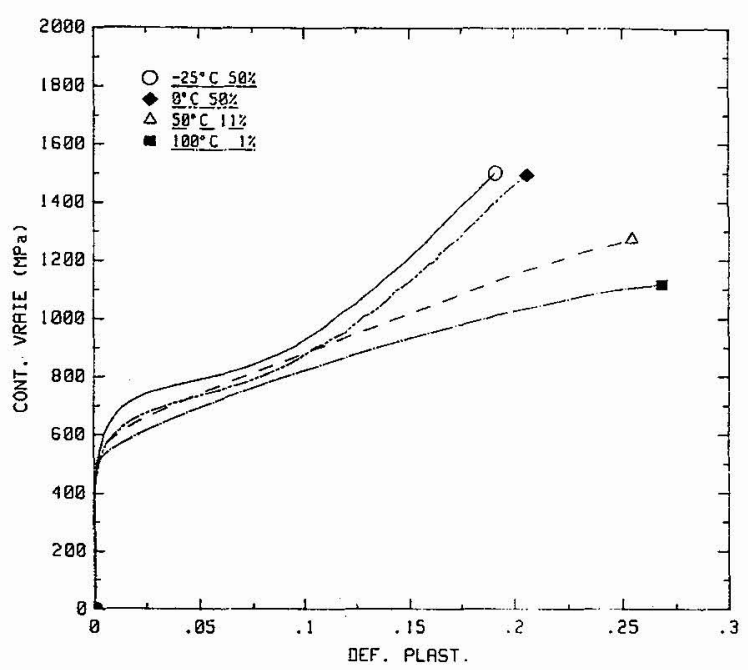

Figure 2 : traction statique à rupture en température de l'acier Z30MCDS1010. Pour chaque température le taux de martensite $\alpha$ formé à rupture est porté.

Il est possible de corréler le relèvement des courbes obtenues pour $0^{\circ} \mathrm{C}$ et $-25^{\circ} \mathrm{C}$ (figure 2) à la forte proportion d'austénite transformée pour ces températures (cf étude microstructurale présentée au 2)). En effet la martensite est plus dure que l'austénite pour ce type d'acier /1/. De plus pour les faibles déformations $(\varepsilon<7 \%$ ) la pente de la courbe de comportement est plus faible aux températures où l'apparition de martensite $\alpha$ ' est significative $\left(0^{\circ} \mathrm{C}\right.$ et $\left.-25^{\circ} \mathrm{C}\right)$ que pour des températures plus élevées ; cet effet est probablement dû à un mécanisme d'orientation de l'écoulement plastique par la transformation à rapprocher du mécanisme de Magee /2/ pour les alliages à mémoire de forme.

\section{2) Etude microstructurale}

Lorsque ces aciers sont soumis à un essai de compression ou de traction statique, une simple microgrảphie (figure 3) révèle l'apparition de plaquettes de martensite dans la structure austénitique initiale.

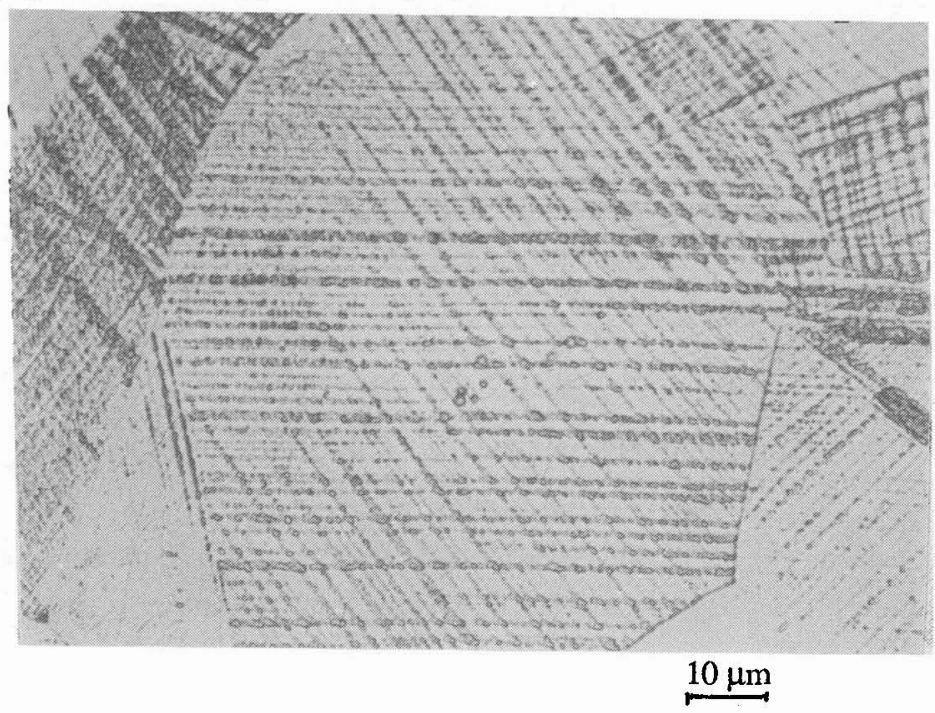

Figure 3 : micrographie de l'acier Z30MCDS1010 ayant subi une compression statique de $5 \%$. 
Une étude au microscope électronique en transmission montre que les deux types possibles de martensites pour cet acier sont présentes : phase $\varepsilon$ héxagonale et phase $\alpha^{\prime}$ quadratique centrée (figure 4 ). On note également la présence de mâcles de déformation et de fautes d'empilement.

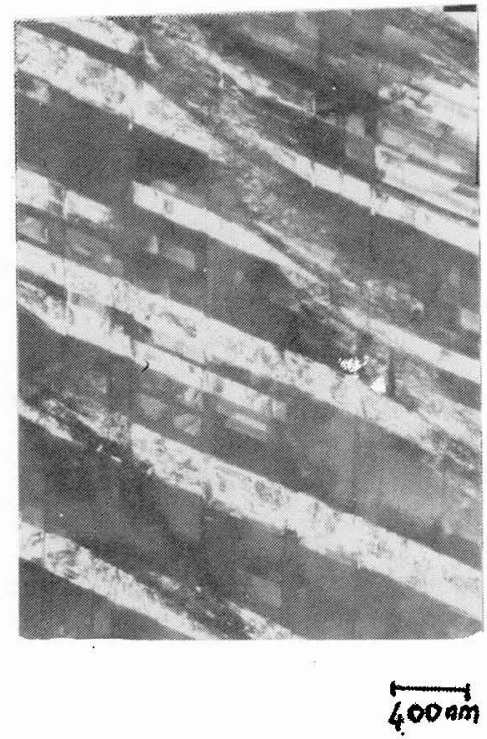

champ sombre

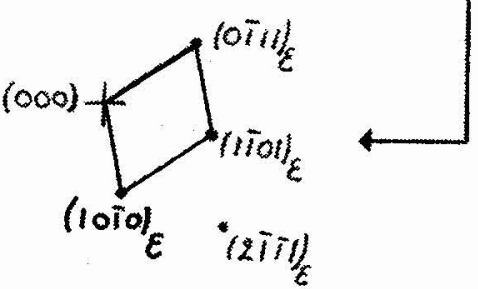

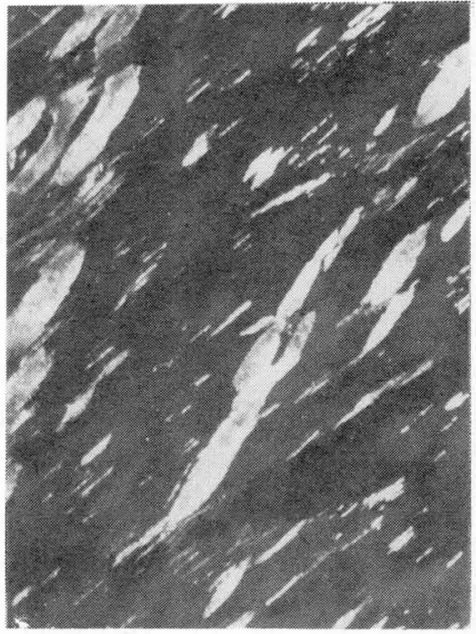

$+$

$500 \mathrm{~mm}$

champ sombre

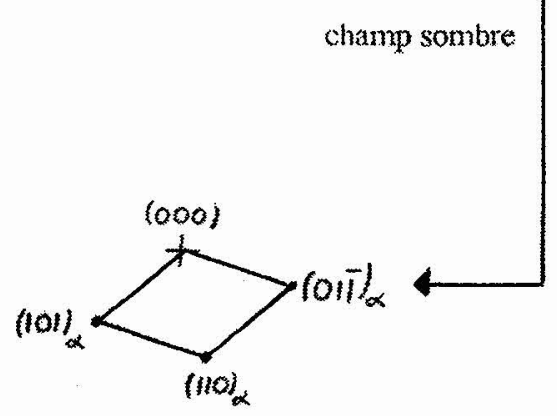

Figure 4 : lattes de martensite $\varepsilon$ à gauche et embryons de martensite $\alpha$ à droite pour des déformations dynamiques de $10 \%$ et $24 \%$ (MET). On observe les mêmes structures que pour les essais statiques.

Ainsi ce matériau présente une nucléation classique de la martensite $\alpha$ ' à l'intersection de "bandes de cisaillement" telles que fautes d'empilement, mâcles de déformation, martensite $\varepsilon$, selon un processus classique pour ce type d'aciers $/ 3 / / 4 /$.

D'autre part, dès $5 \%$ de déformation il semble raisonnable, au vu des clichés de diffraction, de décrire notre matériau comme un matériau biphasé - austénite initiale déformée et martensite $\alpha$ '. Les clichés 
de diffraction ont été réalisés sur des échantillons présentant une texture d'orientation due à la déformation plastique de compression. Le mécanisme de déformation essentiel est lié à la transformation de phase a' et au mouvement des dislocations dans la matrice austénitique.

Cette constatation nous a permis d'interpréter les essais de traction présentés au paragraphe 1) en corrélant l'apparition de la martensite $\alpha^{\prime}$ et l'allure des courbes pour les températures de $-25^{\circ} \mathrm{C}$ et $0^{\circ} \mathrm{C}$.

\section{Essais de compression dynamique}

L'essai dynamique est différent de l'essai statique pour deux raisons principales :

--> La vitesse de déformation est plus élevée ( $\varepsilon=10^{3} \mathrm{~s}^{-1}$ à la barre d'Hopkinson).

--> Il y a échauffement pendant l'essai (la diffusion de la chaleur étant lente par rapport à l'essai).

La conséquence de la première est bien connue : les contraintes d'écoulement sont en moyenne relevées lors d'un essai dynamique en comparaison avec un essai statique (pour un acier n'ayant pas des caractéristiques trop élevées).

La conséquence de la seconde est normalement négligeable pour un acier classique car un échauffement de $100^{\circ} \mathrm{C}$ ne diminue ses caractéristiques que d'au plus $5 \%$. Il en va différemment pour les aciers TRIP. Cela est visible sur la figure 5a où l'on compare l'essai statique à l'essai dynamique dans le cas de l'acier Z30MCDS1010. Pour de faibles déformations, la hiérarchie précédente est respectée car l'élévation de température n'est pas sensible; nous savons d'autre part/4/ que la cinétique de nucléation de la nouvelle phase est plus favorable en dynamique qu'en statique ce qui, pour les faibles déformations, va aussi dans le même sens que la vitesse de déformation. Cependant, dès que la déformation atteint 30\% $c^{\prime}$ est-à-dire pour une élévation moyenne de température de l'ordre de $100^{\circ} \mathrm{C}$ pour un acier classique s'écoulant à $1400 \mathrm{MPa}$ - la tendance est inversée car la thermodynamique de cette transformation solidesolide (austénite-martensite) est d'autant moins favorable que la température augmente (cf figure $5 \mathrm{~b}$ ).
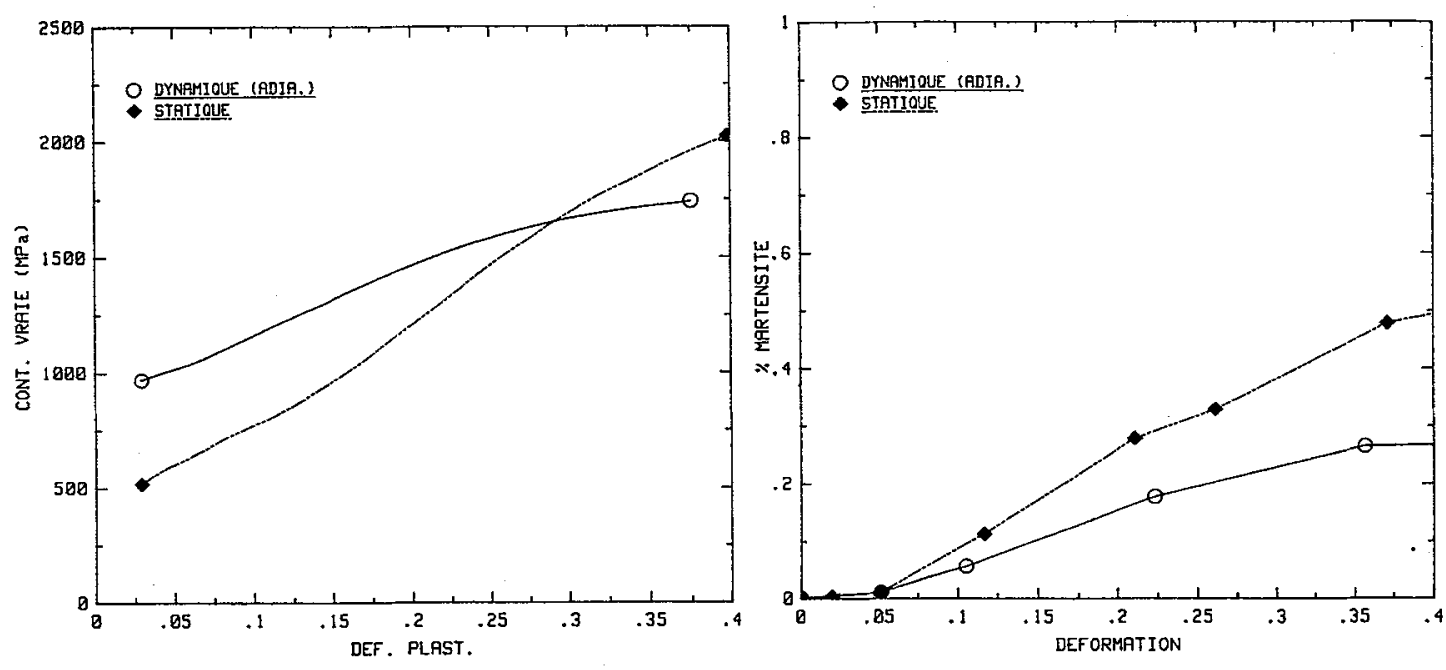

Figure 5a et b : a - comparaison des lois de comportement statique et dynamique à température ambiante dans le cas de la compression de l'acier Ż30MCDS1010 ; b - fractions volumiques de martensite $\alpha$ formées pour les essais de compression statique et dynamique à l'ambiante. 


\section{Etude de l'influence de la vitesse de déformation - essais interrompus}

La remarque précédente peut être précisée ; en effet pour étudier de façon quantitative l'influence de la vitesse de déformation pour ces aciers il convient de limiter la variation de la température pendant l'essai aux barres d'Hopkinson. Pour cela nous avons réalisés la compression d'une éprouvette en limitant la déformation à chaque tir (essais interrompus) de manière à nous affranchir d'une élévation de la température trop importante inhibant la transformation. Sur la figure 6a nous comparons l'essai dynamique "pseudoadiabatique (réalisé en un tir) à l'essai interrompu "pseudo-isotherme ; nous pouvons remarquer que "l'effet TRIP" (remontée de la courbe) est plus marqué dans le cas de l'essai interrompu ; cela est en accord avec la proportion de martensite $\alpha$ formée plus grande dans le cas de l'essai interrompu (figure $6 \mathrm{~b}$ ).
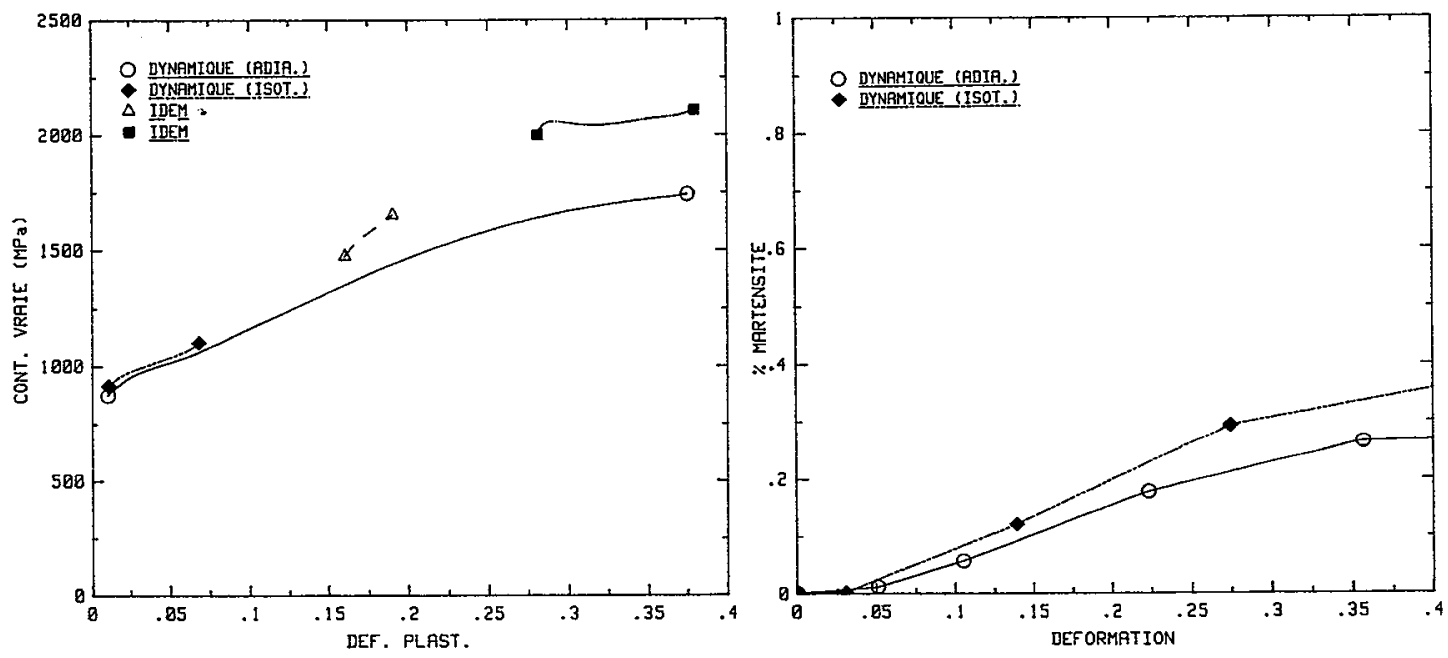

Figure 6a et b : a - comparaison des lois de comportement de l'acier Z30MCDS1010 en compression dynamique depuis la température ambiante pour un essai classique (quasi-adiabatique) et un essai interrompu (quasi-isotherme) ; $b$ - fractions volumiques de martensite $\alpha^{\prime}$ formées correspondantes.

\section{Discussion et perspectives}

L'étude du comportement dynamique de l'acier Z30MCDS1010 présentée au III amène quelques commentaires.

En effet, pour un acier de type Fe-Mn-Cr-C et pour un temps d'application de la charge de l'ordre de 100 $\mu \mathrm{s}$ la transformation martensitique qui s'opère durant l'essai dynamique est de type athermique $/ 6 /$ et de nature adiabatique $/ 7 /$; or il peut être intéressant pour ce type d'acier de calculer l'élévation de température pendant l'essai dynamique à la barre d'Hopkinson - essai non interrompu - pour le corréler quantitativement aux compression statiques effectuées pour différentes températures. Nous supposons que la part de la déformation qui contribue à l'élévation de température et qui est engendrée par le mouvement des dislocations - plasticité classique de l'ausiénite et de la martensite au sens de Leblond /8/ - est transformée à $90 \%$ en chaleur. L'évaluation de la part associée au changement phase est plus délicate pour deux raisons :

La première est que l'on doit être capable d'identifier la part de la déformation totale associée à la transformation $/ 8 /$.

La seconde est qu'il faut pouvoir déterminer la nature plus ou moins exothermique de la transformation ; or cela peut dépendre de la vitesse de croissance de la martensite $/ 7 /$ donc du taux d'élévation de la température pendant l'essai c'est-à-dire de la vitesse de déformation. En somme il faut être 
capable de déterminer la part de déformation stockée par cette déformation de transformation qui ne contribue pas à une élévation de température.

Pour donner un exemple pratique nous avons considéré que $90 \%$ de la déformation anélastique pour l'essai aux barres d'Hopkinson était transformée en chaleur. Cela nous a permis de tracer pour cet essai un graphe température - contrainte (figure 7). D'autre part, pour chaque température, donc chaque déformation, nous avons porté sur le même graphe (figure 7) la contrainte atteinte lors de l'essai statique.

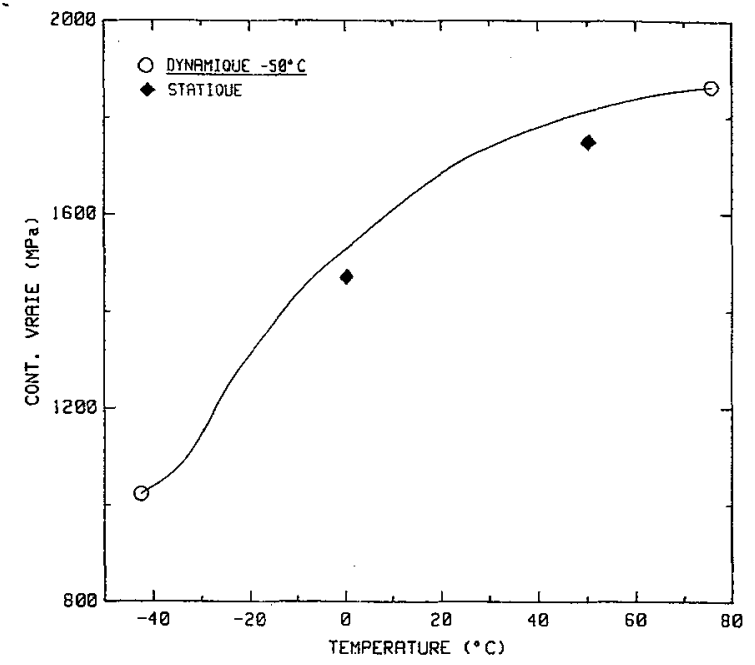

Figure 7 : contrainte en fonction de l'élévation de la température pour un essai dynamique de compression de l'acier Z30MCDS1010 commencé à $-50^{\circ} \mathrm{C}$. Comparaison avec deux essais statiques (même déformation).

L'accord est assez bon mais il reste à déterminer lequel de la germination ou de la crossance est le stade limitant lors de la sollicitation dynamique ; une étude en DSC sous différentes vitesses de refroidissement pourra nous apporter des renseignements supplémentaires. Il reste qu'en statique la forme des courbes $\sigma-\varepsilon$ est analogue à celle des courbes $\% \alpha^{\prime}-\varepsilon$ ce qui n'est pas aussi net en dynamique.

\section{Bibliographie}

/1/ NISHIYAMA, Z., "Martensitic Transformation", Material Science Series, edited by Academic Press, pp $16-48,1978$.

21 MAGEE, C.L., "Transformation Kinetics, Microplasticity and Aging of Martensite in Fe-31Ni", Ph.D. Thesis, Carnegie Institute of Technology, Pittsburgh, PA.

13/ LECROISEY, F. and PINEAU, A., "Martensitic Transformation Induced by Plastic Deformation in the Fe-Ni-Cr-C System", Met. Trans., 3, February 1972, pp.387.

14/ OLSON, C.B. and MORRIS COHEN, "Kinetics of strain-induced martensite nucleation", Met. Trans., 6A, April 1975, pp 791.

15/ STAUDHAMMER, K.P., MURR, L.E. and HECKER, S.S., "Effects of Strain Rate on Deformation-Induced Martensite in 304 Stainless Steel", Shock waves and high-strain-rate phenomena in metals, Edited by M. Meyers and L. E. Murr, 1981.

16/ THADHANI, N.N., MEYERS, M.A., Kinetics of Martensitic Transformation Induced by a Tensile Stress Pulse, Acta Metall., Vol. 34, No. 8, pp. 1625 - 1641, 1986.

n/ NISHIYAMA, Z., "Martensitic Transformation", Material Science Series, Academic Press, edited by M. Fine, M. Meshi, C. Wayman, pp 248-253, 1978.

18/ LEBLOND, J.B., "A Theoretical and Numerical Approach to the Plastic Behaviour of Steels during Phase Transformation - I. Derivation of General Relations", J. Mech. Phys. Solids, Vol. 34, No.4, pp. $395-409,1986$. 